\title{
Design and Implementation of Online Management System for Students' Fourth Class Credits
}

\author{
ZhenMing Chen ${ }^{1, \mathrm{a}}$, Zhengde $\mathrm{Bao}^{1}$ and Chenxi $\mathrm{Li}^{1}$ \\ ${ }^{1}$ Jincheng College of Sichuan University, No.1 Xiyuan Road, High-tech West District, Chengdu, Sichuan Province, China
}

\begin{abstract}
Since the concept of the Fourth Class (Comprehensive quality ability teaching) has been presented, the results of the Fourth Class have been enormous. How to manage information informatization is an urgent problem to be solved. This paper uses the mature management system technology to informatize the Fourth Class management.
\end{abstract}

\section{Introduction}

The Fourth Class (Comprehensive quality ability teaching) of students is a new concept based on the first (Classroom teaching), second (Laboratory teaching) and third (practical teaching) class of students. The Fourth Class of students aims to help students build strong practical ability and increase the conversion rate of knowledge to the results. The Fourth Class of students has the same status as class teaching, and its credits will affect the graduation of students. The Fourth Class encourages students to take an active part in activities and hone themselves. Correspondingly, the record of student participation in the activity and the status of credits are important materials for studying and guiding students in the Fourth Class. However, traditional management methods have numerous problems, such as the lack of student activity records, the confusion of credits, the loss and damage of paper materials, and the opacity of credit information. Therefore, informational means is needed in order to manage the Fourth Class of students. This paper uses the Apache + PHP + MySQL [1] and MVC architecture [2] to develop the Fourth Class management system for students, and realizes the transformation from traditional manual management to informationization.

\section{Analysis and design of online management system for students' Fourth Class credits}

\subsection{System requirements analysis}

The Fourth Class aims to use the extracurricular activities that students love to develop students' professional strengths, develop students' communication expression, organization and coordination, leadership and team spirit, and cultivate students' concern for the country, caring for the society, caring for the people's social responsibility and loving labor. The Fourth Class is divided into three compulsory subjects and nine elective subjects. The Fourth Class encourages students to actively participate in the acquisition of credits through the establishment of activities, competitions, social projects, entrepreneurial projects, art projects, research projects and other practical content.

With the increase in the number of participants in The Fourth Class, the need for student participation analysis, and the management needs of student credits, traditional manual management has revealed major drawbacks. Traditional manual management has the following disadvantages compared to online management systems:

The student's historical participation activities are unknown.

(2) The degree of information transparency is too low

(3) Information sharing between departments is too poor

(4) Historical data is difficult to sort out and analyze

(5) Paper documents are difficult to save, easy to lose

(6) Manual finishing time and labor, easy to make mistakes

(7) Students are difficult to feedback errors in management

Based on the above drawbacks, the Fourth Class management work needs to utilize existing computer resources, utilize the convenience of the network, and develop an online management system based on the existing mature technology [3] to simplify work, improve efficiency, and form a complete and powerful Management system.

\subsection{System goal}

This system is designed for students' Fourth Class credit management. The main objectives are:

(1) Strong management ability for credit certification documents 
(2) History tracking ability of students participating in activities

(3) Management ability of student credits

(4) Processing of student feedback information

(5) Ability to manage student information-

(6) Ability to manage student activities

(7) Ability to organize student's historical information

(8) Different users have different permissions due to the confidentiality of data

(9) The system is stable, safe and reliable

\subsection{System function structure}

According to the system analysis, the system adopts the MVC architecture, and the student application, administrator application, and super administrator application is established on this architecture.

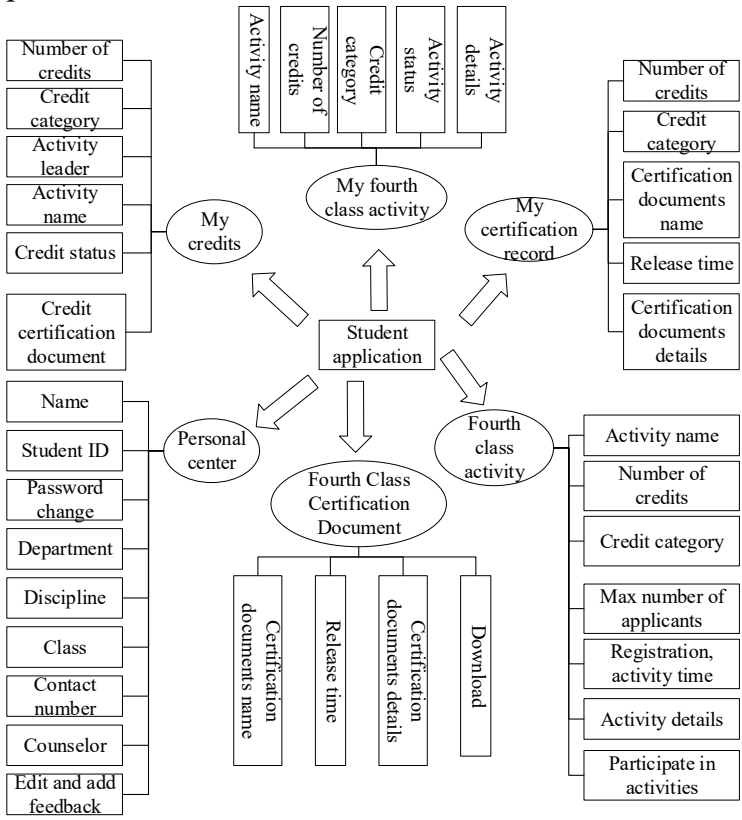

Figure 1. Student application function structure

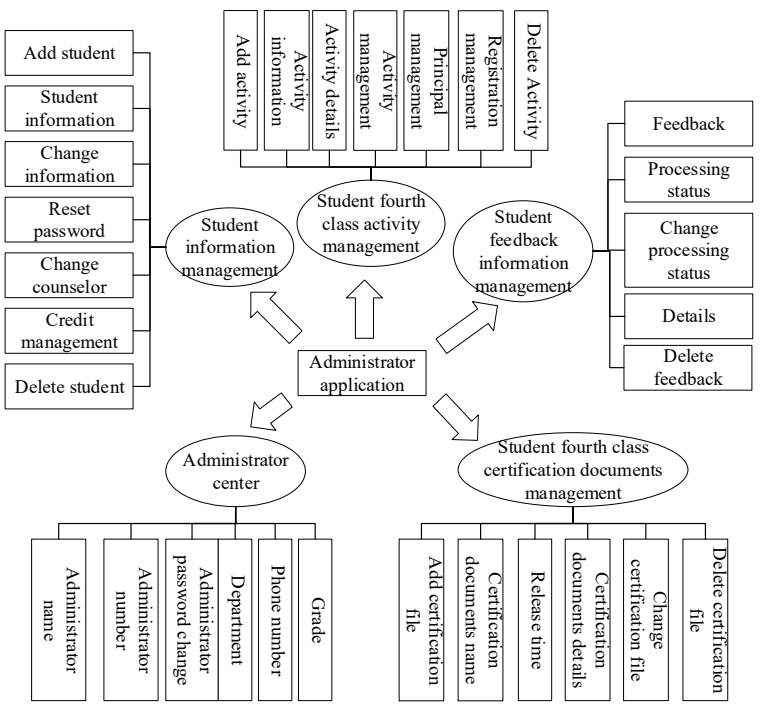

Figure 2. Administrator application structure

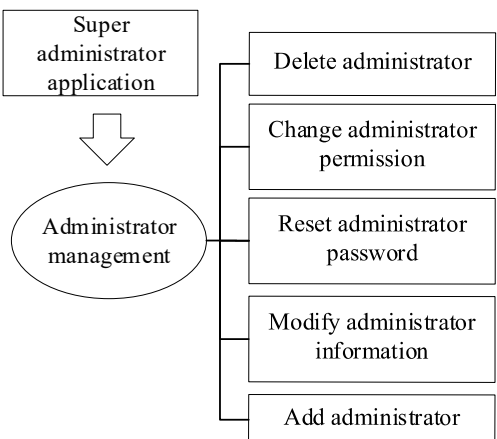

Figure 3. Super administrator application

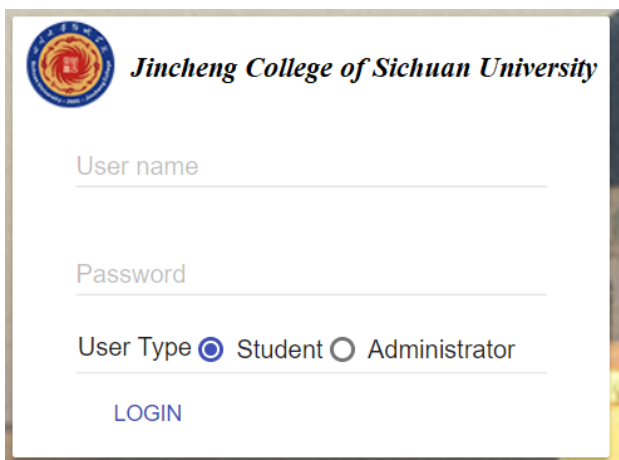

Figure 4. System login interface

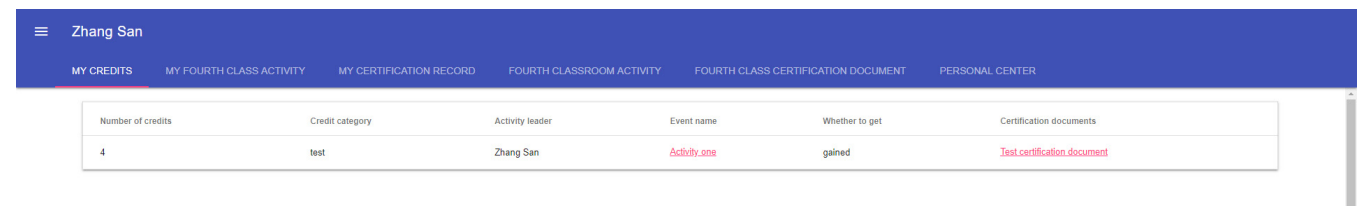

Figure 5. Student application interface

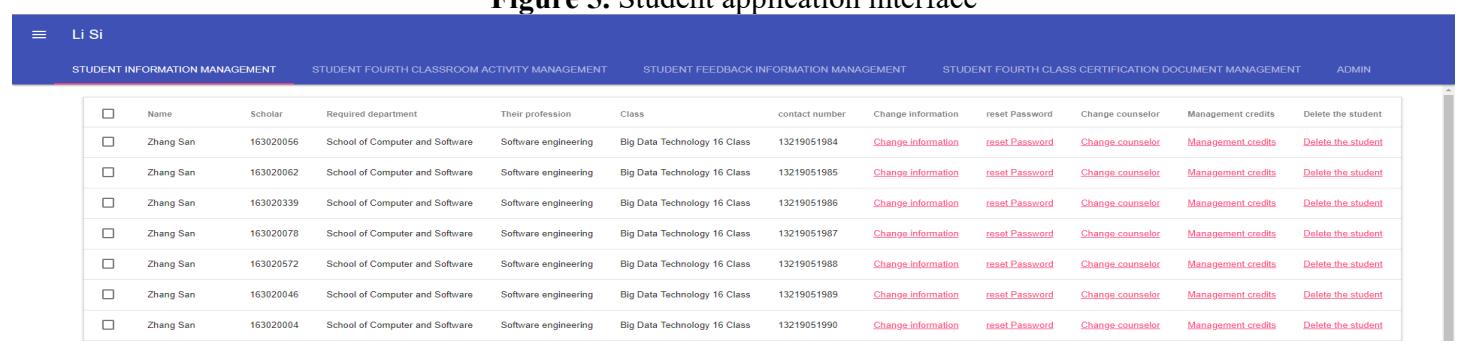

Figure 6. Administrator application interface 


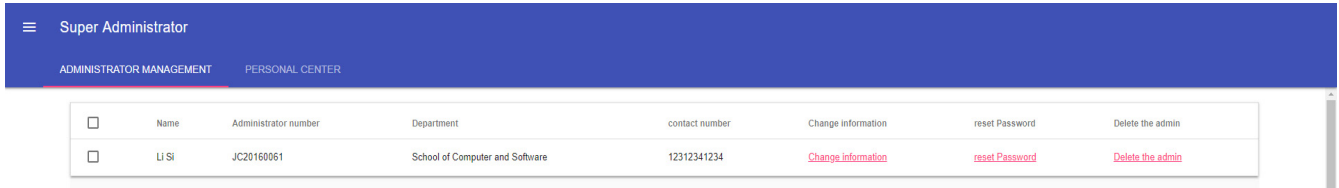

Figure 7. Super admin application interface

\subsection{Database}

On the selection database, the combination of PHP and MySQL is a more common combination[4] in the industry. PHP and MySQL have a tacit understanding and are easy to use. More importantly, MySQL is recognized as an open source database with security, ease of use, efficiency and low cost. The database used in this system is MySQL. In order to have higher compatibility with the system, PDO is used to connect to the database.

\subsubsection{Database concept design}

According to the above requirements analysis and system analysis, the E-R diagram of the entity relationship of the student's Fourth Class online management system is planned out.

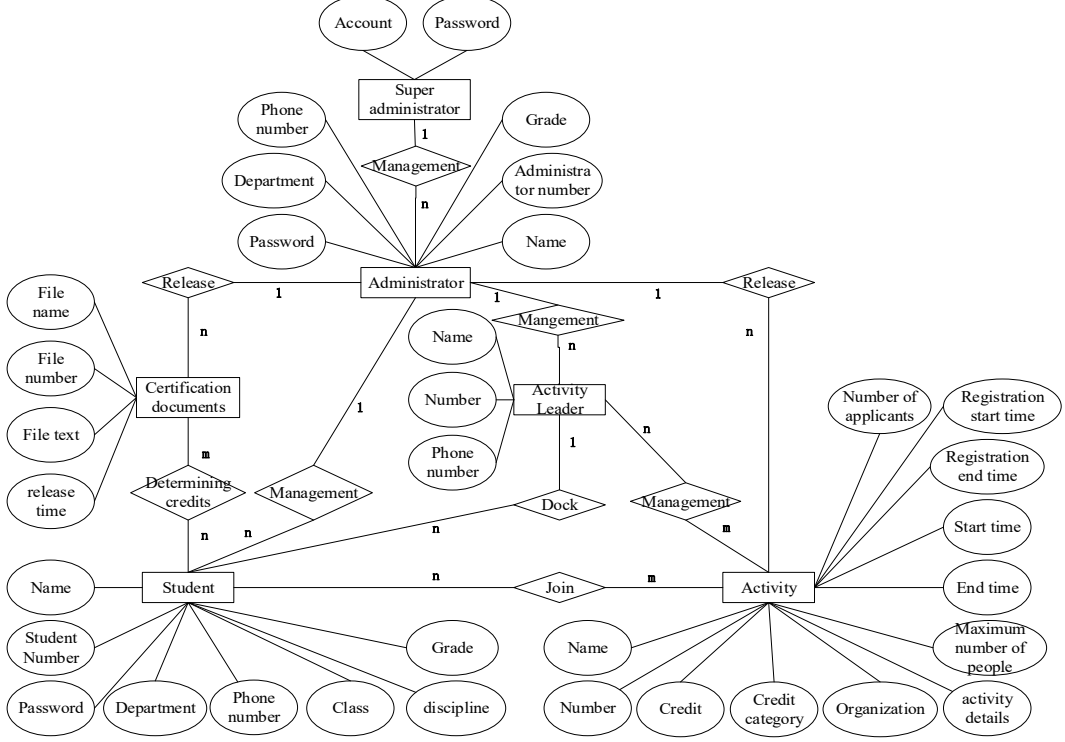

Figure 8. Database E-R

\subsubsection{Database physical structure design}

According to the above database concept design, we created the fourManagementSystem in the database system, which contains 10 data tables, as shown in the figure:

\begin{tabular}{|c|c|c|c|}
\hline Table Name & Type & Sorting rule & Description \\
\hline activity & MyISAM & uft8_unicode_ci & Activity Table \\
\hline activityPrincipal & MyISAM & uft8_unicode_ci & Activity Leader Table \\
\hline activitySignUp & MyISAM & uft8_unicode_ci & Activity Registration Table \\
\hline administrator & MyISAM & uft8_unicode_ci & Administrator Table \\
\hline certificationDocuments & MyISAM & uft8_unicode_ci & Certification Documents Table \\
\hline file & MyISAM & uft8_unicode_ci & File Table \\
\hline studentActivity & MyISAM & uft8_unicode_ci & Student Participation Activities Table \\
\hline studentCredit & MyISAM & uft8_unicode_ci & Credits Table \\
\hline studentFeedback & MyISAM & uft8_unicode_ci & Student Feedback Table \\
\hline studentinformation & MyISAM & uft8_unicode_ci & Student Information Table \\
\hline
\end{tabular}

To simplify the description, only the main data tables are explained.

The Activity Table contains the activity name, activity number, activity credits, credit type, activity attribution organization, maximum number of activities, activity start time, activity end time, activity registration start time, activity registration end time, activity content, and activity registration number. The activity number is the primary key. Its structure is as follows:

\begin{tabular}{|c|c|c|c|c|}
\hline Name & Type & Collation Attributes & S Null Defaul & t. Comments \\
\hline activityName & text & utf8_unicode_ci & No None & Activity name \\
\hline activitylD & varchar (13) & utfi_unicode_ci & No None & Activity number \\
\hline credit & tinyint(4) & & No None & Activity credits \\
\hline creditstatus & varchar(12) & utf__unicode_ci & No None & Credit type \\
\hline activityOrganization & text & utfo_unicode_ci & No None & Activity attribution organization \\
\hline activityMaxPeople & mediumint(9) & & No None & Maximum number of people \\
\hline activityStartTime & $\operatorname{varchar}(10)$ & utf8_unicode_ci & No None & Activity start time \\
\hline activityEndTime & varchar $(10)$ & utfo_unicode_ci & No None & Event end time \\
\hline activitySignUpStartTime & e varchar(10) & utfi_unicode_ci & No None & Event registration start time \\
\hline activitySignUpEndTime & varchar $(10)$ & utfo_unicode_ci & No None & Event registration end time \\
\hline activityText & text & utfo_unicode_ci & No None & Activities \\
\hline activitySignUpNumber & mediumint $(9)$ & & No None & Event registration number \\
\hline
\end{tabular}

Figure 10. Activity Table

The Administrator Table includes the administrator name, administrator number, administrator password, department, mobile phone number, and responsible year level, where the administrator number is the primary key. Its structure is as follows: 


\begin{tabular}{|c|c|c|c|c|c|}
\hline Name & Type & Collation & Attributes Null & Default & t Comments \\
\hline administratororName & varchar $(10)$ & utfo unicode_ci & No & None & Adoministrator name \\
\hline administratotorlD & varchar $(10)$ & utfo_unicode_o & No & None & Aóminstrator number \\
\hline AdministratorPassword & varchar(32) & ) utf__unicode_ci & No & None & Administrator password \\
\hline department & varchar $(10)$ & utfo_unicode_ci & No & None & Deparment \\
\hline phone & varchar(11) & utfo unicode_ $\mathrm{C}$ & No & None & cell phone \\
\hline grade & varchar(2) & ut8_ unicode_ci & No & None & Responsible for the grade \\
\hline
\end{tabular}

The Credits Table includes the student number, the number of credits, the type of credit, the number of the person in charge of the activity, the number of the activity, the number of the certification document, and the type of activity. Its structure is as follows:

\begin{tabular}{|c|c|c|c|c|}
\hline Name & Type Collation & Attributes Null & dll Defaul & it Comments \\
\hline studentle & varchar(9) Uuff_unicode_ci & i No & None & Student number \\
\hline credit & tinyin:(4) & No & None & Number of creats \\
\hline creditstatus & tinyint(1) & No & 0 & Credit type \\
\hline administratortlo & varchar(i1) utf8_unicode_ci & No & None & Activity leader number \\
\hline activityly & varchar(13) utf8_unicode_ci & No & None & Activity number \\
\hline certificationDocumentsliD & varchar(14) uff8_unicode_ci & No & None & Certifcation document numbe \\
\hline activityCategory. & varchar(12) utf8_unicode_ci & No & None & type of activity \\
\hline
\end{tabular}

Figure 12. Credits Table

The student Information Table includes the student's name, student ID number, student password, academic department, student major, mobile phone number, administrator number, student class, and student grade, among which the student number is the primary key. Its structure is as follows:

\begin{tabular}{|c|c|c|c|c|}
\hline Name & Type & Collation & Attributes Null Default & It Comments \\
\hline studentName & $\operatorname{varchar}(10)$ & utfo_unicode_ci & No None & student name \\
\hline studentID & $\operatorname{varchar}(9)$ & utfo_unicode_ci & No None & Student number \\
\hline studentPassword & varchar(32) & utfo_unicode_ci & No None & Student password \\
\hline department & varchar (10) & utfo_unicode_ci & No None & Student deparment: \\
\hline discipline & varchar(32) & utfo_unicode_ci & No None & Student major \\
\hline phone & varchar(11) & utfo_unicode_ci & No None & cell phone \\
\hline administratorlD & varchar (10) & utfo_unicode_ci & No None & Administrator number \\
\hline studentClass & varchar(50) & utfo_unicode_ci & No None & Student class \\
\hline & athatin & $t \rightarrow 0$. & No None & Student grade \\
\hline
\end{tabular}

Figure 13. Student Information

\section{Implementation of the main functions of the system}

\subsection{System architecture implementation}

The Fourth Class Credit Online Management System uses the MVC architecture and builds applications on this architecture.

The system architecture is as follows:

(1) Routing base class: The user request is processed and sent to the controller of the corresponding application.

(2) Model layer base class, view layer base class and controller base class: Their processing of data, operation of the database and view rendering of the result are inherited and extended by each application, and the corresponding functions are added.

(3) Database base class, query base class: The database base class is responsible for accessing the database using different database accounts according to the permissions of the application. The database query base class encapsulates commonly used SQL statements into member functions of the class.

(4) File base class: The file is operated according to the user's permission.

(5) Log base class: Generate corresponding log information when an event occurs.

(6) Verification base class: Filter out unsafe requests.

(7) Analytical base class: Statistical analysis of student historical information, providing a download interface for raw data.

(8) System startup class: After the web server receives the request. It automatically loads the corresponding application and base class.

The system running process is as follows:

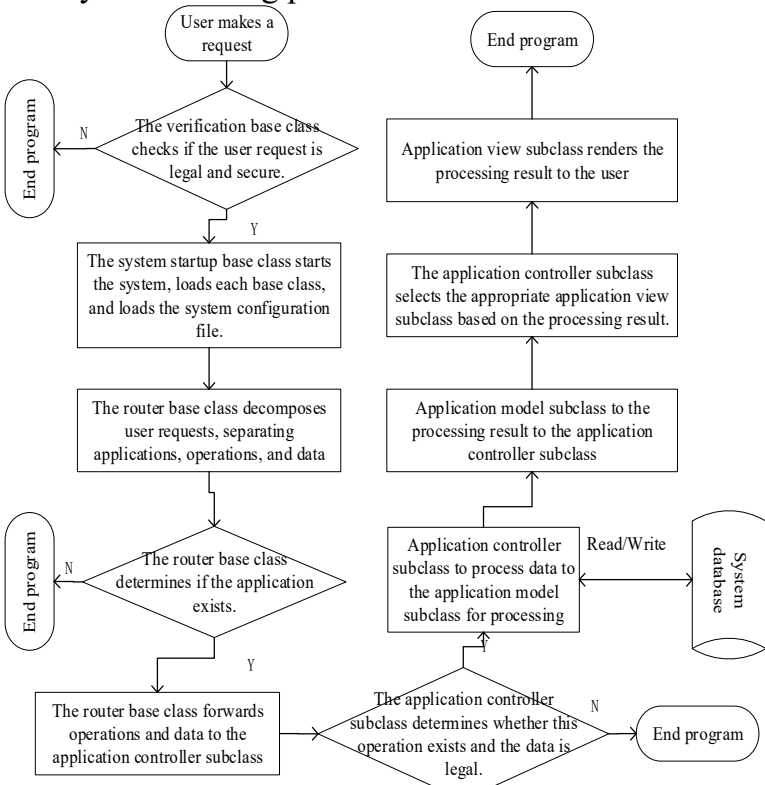

Figure 14. Flow Chart

\subsection{Application implementation}

Due to the large amount of project code, it cannot be fully displayed. This article uses only the simpler super administrator application as an example to demonstrate how to implement the specific application of the system. The super administrator application is a system application for management of Administrator. After the user logs in to the system through the super administrator account, the existing administrator can be managed or a new administrator can be added.

Application view layer uses Google's Material Design Lite framework to list only the necessary code for ease of description.

The main interface frame layout code is as follows: $<$ main $><$ !-- Import views of each function via iframe -- $>$

$<$ section id="super-administrator-main-tab-1">

$<$ iframe

$\mathrm{src}="$ /superAdministrator/administratorInfo" $></$ iframe $><$ $/$ section $>$ 
$<$ section id="super-administrator-main-tab-2" $>$ $<$ iframe

$\mathrm{src}=$ "/superAdministrator/personalCenter" $></$ iframe $></$ s ection $></$ main $>$

Management administrator add administrator function page main code: $<$ form method="post" action="/superAdministrator/administratorInfo" $><\mathrm{t}$ able>

$$
<\text { td }>\text { Name }<\text { input type="text" }
$$

name="administratorName" $></$ td $>$ $<$ td $>$ Administrator $\quad$ Number $<$ input type $=$ "text" name $=$ "administratorID" $></$ td $>$ $<$ td $>$ Department $<$ input type="text" name="department" $></$ td $>$

$$
<\text { td }>\text { Contact Number }<\text { input }
$$

type $=$ "text" name $=$ "phone" $></$ td $>$ $<$ td $>$ Grade $<$ input type="text" name $=$ "grade" $></$ td $>$ $<$ td $><$ input type="submit" name $=$ "submit" value $=$ "add" $></$ td $></$ table $></$ form $>$

Take the example of adding an administrator function, the PHP code is as follows:

// Controller code

class superAdministratorController extends Controller \{

public function administratorInfo(\$administratorID $={ }^{\prime}$, \$type $=$ ', $\$$ data $={ }^{\prime}$ ') \{

// If the parameter is empty, it will be processed according to the form.

if(empty(\$administratorID) \&\& empty(\$type) \&\& isset(\$_POST) \&\& !empty(\$_POST) $\& \&$ ! strcmp('add',\$_POST['submit'])) \{

$$
\$ \text { data }=\text { array }(
$$

'administratorName'

\$_POST['administratorName'],

$$
\text { 'administratorID' }
$$

$=>$

\$_POST['administratorID’], 'administratorPassword' $\quad \Rightarrow$ md5(md5(\$_POST['administratorID'])), 'department'

\$_POST['department'], 'phone' $=>$ \$_POST['phone'], if((new 'grade' $=>$ \$ POST['grade'],);

superAdministrator('administrator'))->addAdministrator( \$data))\{

//Model code

\$logger = Log::getLogger('info');

\$logger->info('Add Administrator'); \$this->show('success'); $\}$ \}\} \}

class superAdministratorModel extends Model \{ // The function responsible for adding administrators public function addAdministrator $(\$$ data $=$ $\operatorname{array}())\{$

if(empty(\$data)) return false;

\$result $=$ this $->$ add $(\$$ data $)$;

if $($ Sresult $>0)$ return true;

else return false; $\}$ \}

\section{Summary}

The Fourth Class Credit Online Management System based on Apache+PHP+MySQL and MVC architecture has the advantages of short development cycle, strong system stability, convenient function expansion and clear business logic. The system can effectively organize the Fourth Class information, enhance the ability of the superintendent to manage the Fourth Class activities and student credits, increase the misorientation and processing ability in the management process, and realize the function of information sharing among multiple departments. It effectively reduces the compliance of managers, simplifies workflow, promotes the informationization process of management tools, and effectively integrates the computer and network resources of colleges and universities.

\section{References}

1. Heqin Zhao. The best solution for designing dynamic websites: Apache + PHP + MySQL $[\mathrm{J}]$. Computer Engineering and Design, 2007(04): 933-934+938.

2. Xiaoli Zhang, Yang Lu. Design and Implementation of Web OA System Based on MVC Pattern[J]. Computer Technology and Development, 2012, 22(08): 63-66.

3. Shun Wang, Yang Zhang, Hua Li, et al. The road to growth of software development engineers: PHP website development practice guide [M]. Tsinghua University Press, 2012.

4. Yang Cui, Yaru He. MySQL database application from entry to the master. 3rd edition [M]. China Railway Publishing House, 2016.

5. Guanghou Zuo. PHP Meditation Record 4 Zend Framework [J]. Programmer, 2007 (12): 100-102. 